\title{
Correction to: Novel CTNNB1-USP6 fusion in intravascular fasciitis of the large vein identified by next-generation sequencing
}

\author{
Ya Lu ${ }^{1} \cdot$ Xin He $^{1} \cdot$ Yan Qiu ${ }^{1} \cdot$ Huijiao Chen ${ }^{1} \cdot$ Hua Zhuang ${ }^{2} \cdot$ Jin Yao ${ }^{3} \cdot$ Hongying Zhang $^{1}$ \\ Published online: 29 May 2020 \\ (C) Springer-Verlag GmbH Germany, part of Springer Nature 2020
}

\section{Correction to: Virchows Archiv \\ https://doi.org/10.1007/s00428-020-02792-x}

The original version of this article, unfortunately, contained errors.

The occurrence of the "exon1 of the CTNNB1" in the line 12, paragraph 3 on the second page of this article should have been changed to "intron1 of the CTNNB1". In Fig. 3c legend the text "transcript" should have been deleted and "the fusion site(arrow) involved exon1 of the CTNNB1 gene" should have been changed to "the break site (arrow) involved intron1 of the CTNNB1 gene". The original paper remains unchanged.

The online version of the original article can be found at https://doi.org/ $10.1007 / \mathrm{s} 00428-020-02792-\mathrm{x}$

\section{Hongying Zhang}

hy_zhang@scu.edu.cn

1 Department of Pathology, West China Hospital, Sichuan University, Guoxuexiang 37, Chengdu 610041, Sichuan, China

2 Department of Ultrasound, West China Hospital, Sichuan University, Chengdu 610041, Sichuan, China

3 Department of Radiology, West China Hospital, Sichuan University, Chengdu 610041, Sichuan, China 